\title{
A pilot study eliminating immunologically-reactive foods from the diet and its effect on symptomatology and quality of life in persons with chronic migraines and headaches
}

\author{
John E. Lewis ${ }^{1 \#}$, Johanna Lopez ${ }^{2}$, Adam Ganuza ${ }^{1}$, Judi M. Woolger ${ }^{3}$, Lawrence Chen ${ }^{1}$, \\ Angelica B. Melillo ${ }^{1}$, Yaima Alonso ${ }^{1}$, Soyona Rafatjah ${ }^{4}$, Janet Konefal ${ }^{4}$, Amine Sarabia ${ }^{1}$, \\ Susanna M. Leonard ${ }^{1}$, Evan G. Long ${ }^{1}$, Eduard Tiozzo ${ }^{1}$ \\ ${ }^{1}$ Department of Psychiatry \& Behavioral Sciences, Miller School of Medicine, University of Miami, Miami, USA \\ ${ }^{2}$ Department of Dietetics and Nutrition, Florida International University, Miami, USA \\ ${ }^{3}$ Department of Medicine, Miller School of Medicine, University of Miami, Miami, USA \\ ${ }^{4}$ Department of Family Medicine \& Community Health, Miller School of Medicine, University of Miami, Miami, USA \\ Email: ;ielewis@miami.edu
}

Received 25 October 2012; revised 28 November 2012; accepted 9 December 2012

\begin{abstract}
Purpose: Chronic migraines and headaches are significant public health problems, and their symptomatologies have been positively linked to diet. We explored if individuals suffering from chronic migraines/ headaches who required medication treatment had improvement in symptomatology and subjective ratings of QoL when following an immune-reactive food exclusion diet based on the results of the ImmunoBloodprint test, an IgG-mediated food sensitivity assay. Methods: Thirty-seven subjects, aged 18 and over, took part in the study. Subjects had to eliminate all reactive foods from their diet for 90 days. Migraine intensity and frequency were measured using the MTAQ, and QoL was assessed with the SF-36 survey at baseline and 30-, 60-, and 90-day follow-up. Results: Subjects who eliminated IgG-mediated reactive foods from their diet had reductions in migraine symptomatology and had improvements in nearly all indicators of QoL, according to the SF-36, from baseline to 90-day follow-up. Conclusions: Subjects were able to improve their migraine symptoms and QoL in response to eliminating IgG reactive foods from the diet. This test may represent a strategy to help mediate chronic migraine symptomatology without the use of medication.
\end{abstract}

Keywords: Migraine Headaches;

Food Sensitivity Testing; Elimination Diet;

Food Intolerance; MTAQ; SF-36

\footnotetext{
*We have no financial or personal disclosures to report regarding the conduct of this research.

${ }^{\#}$ Corresponding author.
}

\section{INTRODUCTION}

Headaches and migraines affect $46 \%$ of the global adult population, and they constitute a significant public health issue given their negative effect on quality of life $(\mathrm{QoL})$ and resulting level of disability [1,2]. Migraines/headaches are multifactorial and involve several different predispositions, the influences of which vary for each individual person. However, many studies positively link diet to symptomatology. Studies have indicated that the percent of patients reporting food as a trigger for migraines range from $12 \%-60 \%$ with many of them reporting more than one food [3-5]. For example, one study evaluated triggers of migraine without aura in 100 subjects and found that in 20 patients migraines were occasionally triggered by foods like chocolate, cheese, wine, tomatoes, nuts, carbonated beverages, and leavened products [6]. Therefore, an individualized diet to relieve migraine is desirable.

At present, the usual method for diagnosing and confirming food hypersensitivity is an elimination diet and food challenge based on patient experience and identification of food triggers [6]. However, this method is labor intensive for both the patient and physician without an objective test to guide food exclusions, since it requires testing all combinations of foods commonly reported by patients to precipitate the symptoms. Previous studies have shown that reducing migraine attacks has been successful via an individualized diet based on the exclusion of immune-reactive foods detected by the presence of Immunoglobulin E (IgE) antibodies [7]. However, symptomatic reactions to food might be caused by another immunologic mechanism, and more recent studies have looked at the role of IgG-mediated responses in food hypersensitivity. IgG antibodies result from a delayed response fol- 
lowing exposure to a particular antigen, contrary to $\operatorname{IgE}$ [8] and are suggested to be causal in some food hypersensitivities [9-11]. For instance, IgG food antibodies may play a role in certain conditions, such as irritable bowel syndrome [12], obesity [13], type I diabetes [14], and migraines. One study among migraine patients tested for the presence of $\operatorname{IgG}$ antibodies to 108 foods with a subsequent elimination diet based on the results. The migraine patients had a significantly higher number of positive results for IgG food allergens than healthy controls, and the food exclusions improved their migraine symptomatology without the use of medication [15]. A randomized, double-blind, crossover trial of an elimination diet based on IgG immune testing found that migraine patients had a significant reduction in the number of headache incidents and days during the food exclusion period [16]. These studies indicate that eliminating IgG-reactive foods may be beneficial for patients by relieving symptoms and improving QoL.

The ImmunoBloodprint is a novel food sensitivity test that detects the presence of IgG antibodies to specific foods. We hypothesized that an individualized elimination diet based on the IgG-reactive foods detected by the ImmunoBloodprint would improve symptoms and $\mathrm{QoL}$ in patients suffering from chronic migraines. Therefore, the purpose of this study was to evaluate changes in headache symptoms, as measured by the Migraine Therapy Assessment Questionnaire (MTAQ), and health-related QoL, as assessed by the SF-36 survey, in patients with chronic migraines and headaches following an immunologically-reactive food elimination diet based on the ImmunoBloodprint results.

\section{METHODS}

\subsection{Subjects}

Subjects $(\mathrm{n}=37)$ who were interested in improving migraine/headache symptomatology and health-related QoL were recruited, screened, and enrolled at the University of Miami Miller School of Medicine between 2008 and 2010. The study was conducted with the approval of the Institutional Review Board for human subjects research, and all participants signed informed consent and HIPAA forms before commencing in the study. The sample comprised of $84 \%$ females $(n=31)$ and $16 \%$ males $(n=6)$ with a mean age of 36.7 years ( $S D=13.1 ; R=20,74)$. The racial/ethnic distribution of the sample was as follows: $51 \%$ white, non-Hispanic $(\mathrm{n}=19), 32 \%$ Hispanic $(\mathrm{n}=12), 11 \%$ black, non-Hispanic $(\mathrm{n}=4)$, and $6 \%$ of other types $(n=2)$. The most prevalent co-morbid diseases and disorders among this sample included: digestive problems $(\mathrm{n}=11)$, low back pain/herniated disc $(\mathrm{n}=$ 10), hypertension $(n=5)$, arthritic conditions $(n=4)$, depression $(\mathrm{n}=3)$, cardiac problems $(\mathrm{n}=3)$, and hepatitis $(\mathrm{n}=3)$. The most prevalent current prescription medications were: anti-inflammatory $(n=7)$, gastrointestinal $(n=$ $6)$, hypertensive $(\mathrm{n}=3)$, dermatologic $(\mathrm{n}=3)$, sleep $(\mathrm{n}=$ $3)$, nutritional $(\mathrm{n}=3)$, and antidepressant $(\mathrm{n}=3)$. The most frequently taken over-the-counter remedies included: Tylenol/Advil/Motrin/Ibuprofen $(\mathrm{n}=22)$, vitamin/ mineral dietary supplement $(\mathrm{n}=16)$, aspirin $(\mathrm{n}=7)$, decongestant $(\mathrm{n}=7)$, antihistamine $(\mathrm{n}=6)$, and antacid ( $=5)$.

\subsection{Study Design}

Potential study candidates were approached and screened among consecutive patients who were being treated at the Department of Medicine clinics and at the Center for Complementary and Integrative Medicine for a wide range of medical and mental health problems. They were identified by the clinicians as potential candidates for migraine headache improvement if they suffered from chronic migraines/headaches of at least one episode per week that required medication treatment. Subjects were excluded from participation if they were: 1) less than 18 years of age; 2) currently participating in another research trial for migraine/headache treatment; 3) suffering from serious medical complications that might limit their participation, such as recent heart attack, stroke, or chronic kidney disease; and/or 4) pregnant. In addition, participants agreed to eliminate reactive foods from their diets for 90 days based on the results of the IgG-mediated test.

Enrolled individuals had their blood drawn by a certified phlebotomist using standard protocols. The blood was sent for processing and analysis to Immuno Laboratories, Inc. (Ft. Lauderdale, FL), which is licensed federally and in several states and accredited by the College of American Pathologists. Immuno Laboratories, Inc. utilizes a proprietary test known as the ImmunoBloodprint that contains microtiter plates with tiny wells that hold antigens for 115 commonly eaten foods and ingredients (see Appendix 1). The participant's blood was tested with each antigen by adding the blood to the microplate, which is then read using laser-like light to precisely detect foods that were reactive to each participant's blood based on $\mathrm{IgG}$ reactions to each antigen.

During the baseline assessment, subjects were provided with the test results and an individualized dietary plan based on the ImmunoBloodprint results, which replaced reactive foods with non-reactive foods. A rotation plan of the non-reactive foods and general information about healthy eating, food preparation, and shopping was given to each participant. No other behaviors were addressed in the recommendations for each participant. The primary advice to each participant was to focus as much as possible on eliminating the reactive foods from the diet for the entire 90-day period. All participants were encouraged to 
contact the study team with questions as they implemented their elimination diet.

\subsection{Outcomes and Assessment Schedule}

Each participant completed a basic demographics and medical history questionnaire at baseline. They were also asked to note any changes in type or amount of their medications during the course of the study. Criteria used to select the assessment instruments included: 1) appropriateness for the population; 2) ease of administration and scoring; and 3) experience administering these measures. The primary outcome of this study was intensity and frequency of migraines/headaches, as measured by the Migraine Therapy Assessment Questionnaire (MTAQ). The MTAQ is a simple, self-report, five-question survey that has been established as a reliable and valid questionnaire to identify migraine sufferers who may benefit from additional care [17]. The secondary outcome was QoL, as measured by the Medical Outcomes Study Short Form-36 (SF-36). The SF-36 provides psychometrically-based physical and mental health summary measures and a preference-based health utility index on physical functioning, physical role functioning, emotional role functioning, mental health, social functioning, vitality, bodily pain, and general health [18]. It is a generic measure that does not target a specific age, disease, or treatment group that provides a t-score for each scale or domain ranging from $0-100$ with higher scores representing better-perceived QoL [18].

Participants also completed a 3-day food record at each assessment to list all food and beverage consumption during that particular time. Subjects recorded their intake on two weekdays and one weekend day prior to the assessment appointment to allow for fluctuations over a normal weekly period. Participants were instructed on how to complete the 3-day food record using common portion sizes and household measures. The 3-day food record was used at each assessment to gauge compliance to the elimination diet based on a comparison of the foods eaten during those three days to the ImmunoBloodprint results of reactive foods for that subject. For example, if a participant ate 20 different foods during the 3-day period and one of the foods was IgG-reactive according to the ImmunoBloodprint results, then the participant was 95\% compliant with the diet for that particular assessment. Participants were assessed on all variables at baseline and 30-, 60-, and 90-day follow-up.

\subsection{Statistical Analysis}

Data were analyzed using SPSS 20 (IBM Inc., Chicago, IL) for Windows. Frequency and descriptive statistics were calculated on all variables. Linear mixed modeling (LMM) was utilized to assess the fixed effect of time on changes in our outcome variables from baseline to the 90-day follow-up period. LMM with heterogeneous compound symmetry covariance allowed us to account for subject attrition, inter-correlated responses between time points, and non-constant variability. If the type III test of the fixed effect of time and the parameter estimate of the baseline to the 90-day fixed effect were significant, then pairwise comparisons were used to determine the unique differences between baseline and follow-up values at 30 , 60 , and 90 days. The criterion for statistical significance was set at $\alpha=0.05$.

\section{RESULTS}

\subsection{Prevalence of IgG Reactive Foods and Dietary Compliance}

For all participants, the average number of IgG-reactive foods and ingredients was $14.3(\mathrm{SD}=6.4)$ with a range of 6 to 34. The top 10 most frequently tested IgG-reactive foods and ingredients were: brewer's yeast (94.6\%), baker's yeast $(89.2 \%)$, wheat $(78.4 \%)$, cow's milk $(64.9 \%)$, egg $(56.8 \%)$, kidney bean $(37.8 \%)$, cheese $(37.8 \%)$, goat's milk (35.1\%), pinto bean (29.7\%), tomato (29.7\%), and mushroom (27.0\%). Average percent compliance to the diet was as follows: 30-day follow-up, 96.6\% ( $\mathrm{SD}=5.5$, $\mathrm{R}=83.3,100)$; 60-day follow-up, $95.1 \%(\mathrm{SD}=6.1, \mathrm{R}=$ $85.7,100)$; and 90-day follow-up, $92.6 \%(\mathrm{SD}=6.1, \mathrm{R}=$ $85.7,100)$.

\subsection{Headache Symptomatology}

Table 1 shows the MTAQ total score at baseline and 30-, 60-, and 90-day follow-up. The MTAQ total score was found to have a marginally significant fixed effect for time $(\mathrm{F}[3,6.2]=3.8, \mathrm{p}=0.08)$, and the parameter estimate between baseline and 90 days was significant ( $\mathrm{t}$ [4.6] $=2.9, \mathrm{p}=0.04)$. Pairwise comparisons revealed that the MTAQ total score at baseline was marginally higher than at 30 days (mean difference $=0.5 ; \mathrm{SE}=0.3 ; 95 \% \mathrm{CI}$ : $-0.02,1.1 ; \mathrm{p}=0.06$ ), at 60 days (mean difference $=0.7$; $\mathrm{SE}=0.3 ; 95 \% \mathrm{CI}:-0.03,1.5 ; \mathrm{p}=0.06)$, and at 90 days (mean difference $=1.1 ; \mathrm{SE}=0.4 ; 95 \% \mathrm{CI}: 0.1,2.2 ; \mathrm{p}=$ $0.04)$.

\subsection{Quality of Life}

Table 1 shows the descriptive values of all eight scales on the SF-36 at baseline and 30-, 60-, and 90-day followup. Our results showed improvement in six of the eight scales (other than mental health and general health). For physical functioning, a significant fixed effect was found for time $(F[3,21.9]=3.6, p=0.03)$, and the parameter estimate between baseline and 90-day follow-up was also significant $(\mathrm{t}[31.0]=-3.1, \mathrm{p}<0.01)$. Pairwise comparisons revealed that physical functioning at baseline 
Table 1. MTAQ and SF-36 scores at baseline and 30, 60, and 90 days.

\begin{tabular}{ccccc}
\hline Measures & Baseline & 30 Days & 60 Days & 90 Days \\
\hline MTAQ Total Score & $3.3 \pm 1.0(1,5)$ & $2.7 \pm 1.1(1,5)$ & $2.5 \pm 1.3(1,5)$ & $2.0 \pm 1.0(1,3)$ \\
SF-36 Physical Functioning & $82.9 \pm 26.8(0,100)$ & $94.2 \pm 9.3(70,100)$ & $89.5 \pm 19.6(35,100)$ & $97.9 \pm 2.7(95,100)$ \\
SF-36 Role-Physical & $79.2 \pm 25.5(0,100)$ & $87.5 \pm 19.4(31.25,100)$ & $84.4 \pm 12.9(62.5,100)$ & $98.2 \pm 4.7(87.5,100)$ \\
SF-36 Role-Emotional & $80.9 \pm 17.2(50,100)$ & $83.3 \pm 22.0(33.3,100)$ & $88.3 \pm 11.2(75,100)$ & $92.9 \pm 10.1(75,100)$ \\
SF-36 Mental Health & $72.1 \pm 16.8(35,100)$ & $71.5 \pm 13.4(45,90)$ & $76.0 \pm 12.2(60,95)$ & $85.7 \pm 5.3(75,90)$ \\
SF-36 Social Functioning & $75.0 \pm 23.6(25,100)$ & $81.7 \pm 18.8(37.5,100)$ & $87.5 \pm 13.2(62.5,100)$ & $98.2 \pm 4.7(87.5,100)$ \\
SF-36 Vitality & $53.9 \pm 19.0(18.75,87.5)$ & $62.5 \pm 12.0(43.75,81.25)$ & $60.0 \pm 20.5(31.25,81.25)$ & $67.9 \pm 11.7(50,81.25)$ \\
SF-36 Bodily Pain & $62.7 \pm 22.3(0,90)$ & $74.9 \pm 13.2(51,90)$ & $77.7 \pm 13.6(51,90)$ & $84.9 \pm 6.4(72,90)$ \\
SF-36 General Health & $63.3 \pm 21.8(15,97)$ & $72.4 \pm 16.0(47,97)$ & $68.8 \pm 20.5(37,100)$ & $74.3 \pm 17.6(47,100)$ \\
\hline
\end{tabular}

Note: Values are mean \pm standard deviation (minimum, maximum). Sample size for MTAQ: Baseline $(\mathrm{n}=30), 30$ Days $(\mathrm{n}=11)$, 60 Days $(\mathrm{n}=8)$, and 90 Days $(\mathrm{n}=5)$. Sample size for SF-36: Baseline $(\mathrm{n}=34), 30$ Days $(\mathrm{n}=13), 60$ Days $(\mathrm{n}=10)$, and 90 Days $(\mathrm{n}=7)$.

was significantly lower than at 30 days (mean difference $=-8.7 ; \mathrm{SE}=3.9 ; 95 \% \mathrm{CI}:-16.8,-0.7 ; \mathrm{p}=0.04)$ and at 90 days (mean difference $=-13.4 ; \mathrm{SE}=4.3 ; 95 \% \mathrm{CI}$ : $-22.1,-4.6 ; \mathrm{p}<0.01)$, but not at 60 days. For role-physical, a significant fixed effect was found for time $(\mathrm{F}[3,21.5]=8.3, \mathrm{p}<0.01)$, and the parameter estimate between baseline and 90-day follow-up was also significant $(\mathrm{t}[35.1]=-4.4, \mathrm{p}<0.001)$. Pairwise comparisons revealed that role-physical was significantly higher at 90 days than at baseline (mean difference $=17.8$; $\mathrm{SE}=4.0 ; 95 \% \mathrm{CI}: 9.7,25.9 ; \mathrm{p}<0.001$ ), 30 days (mean difference $=11.4 ; \mathrm{SE}=4.9 ; 95 \% \mathrm{CI}: 0.8,22.1 ; \mathrm{p}<0.05)$, and 60 days (mean difference $=12.5 ; \mathrm{SE}=3.6 ; 95 \% \mathrm{CI}$ : $4.9,20.2 ; \mathrm{p}<0.01)$. For role-emotional, the fixed effect for time was non-significant $(\mathrm{F}[3,18.3]=2.2, \mathrm{p}=0.12)$, but the parameter estimate between baseline and 90-day follow-up was significant $(\mathrm{t}[11.3]=-2.4, \mathrm{p}=0.03)$. Pairwise comparisons revealed that role-emotional significantly improved from baseline to 90 days (mean difference $=-10.5 ; \mathrm{SE}=4.4 ; 95 \% \mathrm{CI}:-20.1,-1.0 ; \mathrm{p}=$ 0.03 ), but not at 30 or 60 days. For social functioning, a significant fixed effect was found for time $(\mathrm{F}[3,22.7]=$ $9.7, \mathrm{p}<0.001)$, and the parameter estimate between baseline and 90-day follow-up was also significant $(\mathrm{t}$ [36.5] $=$ $-5.1, \mathrm{p}<0.001)$. Pairwise comparisons revealed that social functioning was significantly higher at 90 days than at baseline (mean difference $=20.2 ; \mathrm{SE}=3.9 ; 95 \% \mathrm{CI}$ : $12.2,28.1 ; \mathrm{p}<0.001), 30$ days (mean difference $=14.8$; $\mathrm{SE}=4.4 ; 95 \% \mathrm{CI}: 5.4,24.3 ; \mathrm{p}<0.01)$, and 60 days (mean difference $=8.9 ; \mathrm{SE}=3.7 ; 95 \% \mathrm{CI}: 1.0,16.9 ; \mathrm{p}<$ $0.05)$. For vitality, a significant fixed effect was found for time $(\mathrm{F}[3,21.0]=5.2, \mathrm{p}<0.01)$, and the parameter estimate between baseline and 90-day follow-up was also significant $(\mathrm{t}[18.5]=-3.9, \mathrm{p}=0.001)$. Pairwise comparisons revealed that vitality had improved at 90 days compared to baseline (mean difference $=16.3 ; \mathrm{SE}=4.2$; 95\% CI: 7.5, 25.2; $\mathrm{p}=0.001$ ), but not at 30 and 60 days. For bodily pain, a significant fixed effect was found for time $(\mathrm{F}[3,27.9]=10.4, \mathrm{p}<0.001)$, and the parameter estimate between baseline and 90-day follow-up was also significant $(\mathrm{t}[36.5]=-5.4, \mathrm{p}<0.001)$. Pairwise comparisons revealed that bodily pain had improved at 90 days compared to baseline (mean difference $=20.5 ; \mathrm{SE}=3.8$; 95\% CI: 12.8, 28.2; p < 0.001), 30 days (mean difference $=11.0 ; \mathrm{SE}=3.3 ; 95 \% \mathrm{CI}: 4.0,17.9 ; \mathrm{p}<0.01)$, and 60 days (mean difference $=8.0 ; \mathrm{SE}=3.8 ; 95 \% \mathrm{CI}$ : 0.3, 15.6; $\mathrm{p}<0.05)$.

\section{DISCUSSION}

Conventional treatment of migraines and headaches is aimed at symptom resolution, so improvement in QoL is the most significant benefit for a patient with this chronic condition. In this clinic-based study, patients with chronic migraines/headaches were prescribed a food exclusion diet based on the presence of $\operatorname{IgG}$, as detected by the ImmunoBloodprint test. The results of this study indicate that our subjects experienced clinically significant improvements in migraine/headache symptomatology and QoL, as measured by the MTAQ and the SF-36. Migraine frequency and intensity did not show as strong a reduction as the broader outcomes of overall QoL. Changes in SF-36 scores were observed in physical functioning, role-physical, role-emotional, social functioning, vitality, and bodily pain, indicating an extensive array of subjective QoL improvements. Thus, the MTAQ and the SF-36 indicated reasonable improvements in migraine/headache symptomatology and QoL in response to an IgG-mediated food elimination diet.

Previous data have shown that the effects of the elimination diet on QoL are beneficial, but the mechanisms 
through which diet affects migraine are unclear. Although IgE testing is the most commonly recognized method to determine food hypersensitivity $[19,20]$, some researchers have suggested that non-IgE mechanisms are involved in the symptomatic reaction to certain foods [21]. The IgG antibody response may be one of those mechanisms of food hypersensitivity $[22,23]$. Despite the potential usefulness of $\operatorname{IgG}$ testing as a clinical tool, the medical community does not universally accept it, since IgG has been found in healthy individuals after ingestion of foods [24-26]. It is unknown what percentage of the population with high levels of $\mathrm{IgG}$ food-related antibodies has no migraine/headache symptoms. In addition, interlaboratory reproducibility is lacking, and the sensitivity and specificity of $\operatorname{IgG}$ food hypersensitivity testing need to be evaluated [23].

Our study, among others, demonstrates that eliminating foods that are IgG-reactive, while replacing them with similar, non-reactive foods to prevent nutrient deficiencies, is a novel strategy for addressing chronic migraines/ headaches. IgG testing directs the identification of reactive foods, minimizing the taxing trial and error required of oral food challenge. Dietary food challenge is considered the only modality for truly identifying clinical reaction to foods [27,28], but both practitioner and patient can benefit from cues taken from IgG reactivity screening.

Studies employing blinded food challenges are also necessary to corroborate $\operatorname{IgG}$ testing results and thus aid in overcoming the bias against this potentially useful tool. Even though our study did not employ food challenge after the elimination diet to identify the foods that result in true clinical outcomes, our results provide evidence that the ImmunoBloodprint could be effective in guiding exclusion diets for this purpose. Hence, further studies are warranted in which ImmunoBloodprint tests are used to guide exclusion diets and food challenges.

\section{Limitations}

Limitations of this study include the lack of any additional biological markers of inflammation, e.g., C-reactive protein, cytokines, or growth factors, to be able to determine the possible relationship between changes in migraine/headache symptoms and QoL with indicators of chronic inflammation. The study is limited by the small sample size and the lack of a control group of participants with chronic migraines on a "sham" diet, thus the results of the study should be interpreted accordingly. While we utilized a small sample, we excluded individuals currently undergoing other interventions for migraines concurrent to the study to at least partially minimize confounding factors. While our study is not large enough to provide conclusive evidence that eliminating IgGreactive foods can completely eliminate migraines and chronic headaches, it does serve to: 1) establish a statis- tically-significant link between ImmunoBloodprint guided diets and positive change in migraine symptoms and 2) show that a larger, more comprehensive study is warranted. Previous food elimination diets have reported that patients on sham diets also improved to a lesser extent on QoL measures, highlighting the importance of performing double-blind, randomized controlled trials in order to prevent overestimation of the potential benefit of the intervention [23]. Furthermore, the measures we utilized are subjective, patient self-reported. However, the validity of the MTAQ and SF-36 are well-established, and their efficacy was one of the primary reasons they were selected for use in the study. Migraines and chronic headaches are inherently difficult to objectively quantify. The MTAQ is concise, effective, and symptom specific. Lastly, participants were not re-assessed with another IgG test at 90 days to determine consistency across the study and/or to denote any changes in response to the intervention.

\section{CONCLUSION}

The results of the study suggest that the IgG-mediated ImmunoBloodprint test for food hypersensitivity can be a useful tool in identifying foods to be excluded from the diet of individuals suffering from chronic migraines/headaches. The exclusion of these foods leads to significant improvement in symptoms and QoL measures over a 90day period. Regardless of the exact mechanism in which migraines are triggered, the individualized exclusion diets outlined in this study prove to be a simple, safe, and costeffective method to control the often times debilitating effects of chronic migraines. IgG-mediated tests and the individualized exclusion diets based on their results are a tool and healthcare strategy that merit further clinical and biomedical research.

\section{ACKNOWLEDGEMENTS}

We are thankful to all the volunteers who participated in this study. This study was supported by a gift from Immuno Laboratories of Ft. Lauderdale, FL.

\section{REFERENCES}

[1] Bussone, G., Usai, S., Grazzi, L., Rigamonti, A., Solari, A. and D'Amico, D. (2004) Disability and quality of life in different primary headaches: Results from Italian studies. Neurological Science, 25, S105-S107. doi:10.1007/s10072-004-0263-y

[2] Stovner, L., Hagen, K., Jensen, R., Katsarava, Z., Lipton, R., Scher, A., Steiner, T. and Zwart, J.A. (2007) The global burden of headache: A documentation of headache prevalence and disability worldwide. Cephalalgia, 27, 193210. doi:10.1111/j.1468-2982.2007.01288.x

[3] Andress-Rothrock, D., King, W. and Rothrock, J. (2010) An analysis of migraine triggers in a clinic-based popula- 
tion. Headache, 50, 1366-1370. doi:10.1111/j.1526-4610.2010.01753.x

[4] Carod-Artal, F.J., Ezpeleta, D., Martin-Barriga, M.L. and Guerrero, A.L. (2011) Triggers, symptoms, and treatment in two populations of migraneurs in Brazil and Spain. A cross-cultural study. Journal of Neurological Science, 304, 25-28. doi:10.1016/i.jns.2011.02.027

[5] Kelman, L. (2007) The triggers or precipitants of the acute migraine attack. Cephalalgia, 27, 394-402. doi:10.1111/j.1468-2982.2007.01303.x

[6] Finocchi, C. and Sivori, G. (2012) Food as trigger and aggravating factor of migraine. Neurological Science, 33, 77-80. doi:10.1007/s10072-012-1046-5

[7] Mansfield, L.E., Vaughan, T.R., Waller, S.F., Haverly, R.W. and Ting, S. (1985) Food allergy and adult migraine: Double-blind and mediator confirmation of an allergic etiology. Annals of Allergy, 55, 126-129.

[8] Crowe, S.E. and Perdue, M.H. (1992) Gastrointestinal food hypersensitivity: Basic mechanisms of pathophysiology. Gastroenterology, 103, 1075-1095.

[9] el Rafei, A., Peters, S.M., Harris, N. and Bellanti, J.A. (1989) Diagnostic value of IgG4 measurements in patients with food allergy. Annals of Allergy, 62, 94-99.

[10] Host, A., Husby, S., Gjesing, B., Larsen, J.N. and Lowenstein, H. (1992) Prospective estimation of IgG, IgG subclass and IgE antibodies to dietary proteins in infants with cow milk allergy. Levels of antibodies to whole milk protein, BLG and ovalbumin in relation to repeated milk challenge and clinical course of cow milk allergy. Allergy, 47, 218-229. doi:10.1111/j.1398-9995.1992.tb00654.x

[11] Awazuhara, H., Kawai, H. and Maruchi, N. (1997) Major allergens in soybean and clinical significance of IgG4 antibodies investigated by IgE- and IgG4-immunoblotting with sera from soybean-sensitive patients. Clinical and Experimental Allergy, 27, 325-332. doi:10.1111/j.1365-2222.1997.tb00711.x

[12] Finn, R., Smith, M.A., Youngs, G.R., Chew, D., Johnson, P.M. and Barnes, R.M. (1987) Immunological hypersensitivity to environmental antigens in the irritable bowel syndrome. The British Journal of Clinical Practice, 41, 1041-1043.

[13] Wilders-Truschnig, M., Mangge, H., Lieners, C., Gruber, H., Mayer, C. and Marz, W. (2008) IgG antibodies against food antigens are correlated with inflammation and intima media thickness in obese juveniles. Experimental and Clinical Endocrinology \& Diabetes, 116, 241-245. doi:10.1055/s-2007-993165

[14] Kohno, T., Kobashiri, Y., Sugie, Y., Takai, S., Watabe, K., Kaino, Y. and Kida, K. (2002) Antibodies to food antigens in Japanese patients with type 1 diabetes mellitus. Diabetes Research and Clinical Practice, 55, 1-9. doi:10.1016/S0168-8227(01)00250-9

[15] Arroyave H.C.M., Echevarria P.M. and Hernandez M.H.L. (2007) Food allergy mediated by IgG antibodies associated with migraine in adults. Revista Alergia Mexico, 54, 162-168.

[16] Alpay, K., Ertas, M., Orhan, E.K., Ustay, D.K., Lieners, C. and Baykan, B. (2010) Diet restriction in migraine, based on IgG against foods: A clinical double-blind, ran- domised, cross-over trial. Cephalalgia, 30, 829-837. doi: $10.1177 / 0333102410361404$

[17] Chatterton, M.L., Lofland, J.H., Shechter, A., Curtice, W.S., Hu, X.H., Lenow, J., Smullens, S.N., Nash, D.B. and Silberstein, S.D. (2002) Reliability and validity of the migraine therapy assessment questionnaire. Headache, 42, 1006-1015. doi:10.1046/j.1526-4610.2002.02230.x

[18] Ware, J., Kosinski, M. and Dewey, J. (2000) How to score version two of the sf-36 health survey. QualityMetric, Incorporated, Lincoln.

[19] Asero, R., Ballmer-Weber, B.K., Beyer, K., Conti, A., Dubakiene, R., Fernandez-Rivas, M., Hoffmann-Sommergruber, K., Lidholm, J., Mustakov, T., Oude Elberink, J.N., Pumphrey, R.S., Stahl, S.P., Ree van, R., Vlieg-Boerstra, B.J., Hiller, R., Hourihane, J.O., Kowalski, M., Papadopoulos, N.G., Wal, J.M., Mills, E.N. and Vieths, S. (2007) IgE-mediated food allergy diagnosis: Current status and new perspectives. Molecular Nutrition \& Food Research, 51, 135-147. doi:10.1002/mnfr.200600132

[20] Ortolani, C., Bruijnzeel-Koomen, C., Bengtsson, U., Bindslev-Jensen, C., Bjorksten, B., Host, A., Ispano, M., Jarish, R., Madsen, C., Nekam, K., Paganelli, R., Poulsen, L.K. and Wuthrich, B. (1999) Controversial aspects of adverse reactions to food. European Academy of Allergology and Clinical Immunology (EAACI) Reactions to Food Subcommittee. Allergy, 54, 27-45. doi:10.1034/j.1398-9995.1999.00913.x

[21] Halpern, G.M. and Scott, J.R. (1987) Non-IgE antibody mediated mechanisms in food allergy. Annals of Allergy, 58, 14-27.

[22] Atkinson, W., Sheldon, T.A., Shaath, N. and Whorwell, P.J. (2004) Food elimination based on IgG antibodies in irritable bowel syndrome: A randomised controlled trial. Gut, 53, 1459-1464. doi:10.1136/gut.2003.037697

[23] Drisko, J., Bischoff, B., Hall, M. and McCallum, R. (2006) Treating irritable bowel syndrome with a food elimination diet followed by food challenge and probiotics. Journal of the American College of Nutrition, 25, 514-522.

[24] Husby, S., Oxelius, V.A., Teisner, B., Jensenius, J.C. and Svehag, S.E. (1985) Humoral immunity to dietary antigens in healthy adults. Occurrence, isotype and IgG subclass distribution of serum antibodies to protein antigens. International Archives of Allergy and Applied Immunology, 77, 416-422. doi:10.1159/000233819

[25] King, H.C. (1994) Exploring the maze of adverse reactions to foods. Ear, Nose \& Throat Journal, 73, 237-241.

[26] Kruszewski, J., Raczka, A., Klos, M. and Wiktor-Jedrzejczak, W. (1994) High serum levels of allergen specific IgG4 (asIgG-4) for common food allergens in healthy blood donors. Archivum Immunologiae et Therapiae Experimentalis (Warsz), 42, 259-261.

[27] Sicherer, S.H. (2001) Clinical implications of cross-reactive food allergens. Journal of Allergy and Clinical Immunology, 108, 881-890. doi:10.1067/mai.2001.118515

[28] Sicherer, S.H. (1999) Food allergy: When and how to perform oral food challenges. Pediatric Allergy and Immunology, 10, 226-234. doi:10.1034/j.1399-3038.1999.00040.x 


\section{APPENDIX 1}

The 115 tested foods comprising the ImmunoBloodprint.

\begin{tabular}{|c|c|c|c|c|}
\hline Alfalfa & Celery & Herring & Parsley & Seed, Rape \\
\hline Almond & Cheese & Lamb & Pea & Canola \\
\hline Amaranth & Cherry & Lemon & Peach & Sesame \\
\hline Apple & Chicken & Lentil & Peanut & Shrimp \\
\hline Asparagus & Cinnamon & Lettuce & Pecan & Snapper \\
\hline Avocado & Clam & Lime & Pepper, B/W & Sole \\
\hline Banana & Clove & Lobster & Pepper, Chili & Soybean \\
\hline Barley & Cocoa-Chocolate & Mackerel & $\begin{array}{c}\text { Pepper, } \\
\text { Green }\end{array}$ & Spinach \\
\hline Bean, Green & Coconut & $\begin{array}{l}\text { Milk, } \\
\text { Cow's }\end{array}$ & Perch & Strawberry \\
\hline Bean, Kidney & Cod & $\begin{array}{l}\text { Milk, } \\
\text { Goat's }\end{array}$ & Pineapple & Sugar, Cane \\
\hline Bean, Lima & Coffee & Millet & Plum & Sunflower \\
\hline Bean, Mung & Corn & Mushrooms & Pork & Tangerine \\
\hline Bean, Pinto & Crab & Mustard & Potato, Sweet & Tea \\
\hline $\begin{array}{c}\text { Bean, Yellow } \\
\text { Wax }\end{array}$ & Cranberry & Nut, Brazil & Potato, White & Tomato \\
\hline Beef & Egg & $\begin{array}{l}\text { Nut, } \\
\text { Cashew }\end{array}$ & Pumpkin & Trout \\
\hline Beet & Eggplant & Nutmeg & Quinoa & Tuna \\
\hline Broccoli & Flounder & Oat & Radish & Turkey \\
\hline $\begin{array}{l}\text { Brussels } \\
\text { Sprouts }\end{array}$ & Garlic & Olive & Rice & Walnut \\
\hline Buckwheat & Ginger & Onion & Rye & Wheat \\
\hline Cabbage & Grape & Orange & Safflower & White Fish \\
\hline Cantaloupe & Grapefruit & Oregano & Sage & Yam \\
\hline Carrot & Haddock & Oyster & Salmon & $\begin{array}{l}\text { Yeast, } \\
\text { Baker's }\end{array}$ \\
\hline \multirow[t]{2}{*}{ Cauliflower } & Halibut & Papaya & Scallops & $\begin{array}{c}\text { Yeast, } \\
\text { Brewer's }\end{array}$ \\
\hline & & & & Zucchini \\
\hline
\end{tabular}

\section{ABBREVIATIONS}

Immunoglobulin $\mathrm{G}(\mathrm{IgG})$

Migraine Therapy Assessment Questionnaire (MTAQ)

Short-Form Health Survey 36 (SF-36)

Quality of Life (QoL)

Immunoglobulin E (IgE)

Standard Deviation (SD)

Statistical Package for Social Sciences 20 (SPSS 20)

Linear Mixed Modeling (LMM)

Confidence Interval (CI)

Standard Error (SE) 